\title{
Teoria das Probabilidades e Cadeias de Markov.
}

\section{Marianna Degani*, Francys Souza, Paulo Ruffino. \\ Resumo}

Nesse projeto, caracterizamos a probabilidade segundo os axiomas de Kolmogorov [3]. Começamos com as ideias "básicas" (teoria dos conjuntos, $\sigma$-álgebra e $\sigma$-álgebra de Borel) a partir do qual evoluímos para os conceitos de variável aleatória, esperança vista como integral de Lebesgue [2], Teorema de Radon-Nikodym, esperança condicional e os teoremas limites. Na segunda parte do projeto, caracterizamos filtragem, e processos estocásticos. Nos concentrando em cadeias de Markov [1] no qual estudamos conceitos como tempos de parada, estados de uma cadeia de Markov, probabilidades de transição, autovalores, cadeias irredutíveis e comportamento limite de uma cadeia de Markov.

\section{Palavras-chave:}

Probabilidade, $\sigma$-algebra, Cadeias de Markov.

\section{Introdução}

A probabilidade é o ato de atribuirmos "pesos" aos eventos, mas a qual eventos vamos atribuir probabilidade?

Para responder essa questão adotamos a definição proposta por Kolmogorov (1932) de uma consequência da construção axiomática de probabilidade. Também definimos a integral de Lebesgue, a esperança condicional e o teorema de Radon-Nikodym representando um funcional linear sobre um espaço de Hilbert.

\section{Resultados e Discussão}

Definição 1.1: Um experimento com espaço amostral $\Omega$ e classe de eventos A. Além disso, a probabilidade deve satisfazer os seguintes axiomas:

- $P(\Omega)=1$ e $P(\varnothing)=0$.

- $0 \leq ?(A) \leq 1$, para todo evento $\mathrm{A}$.

- Para qualquer sequência de eventos mutuamente exclusivos $A_{1}, A_{2}, \ldots$, isto é, eventos

para os quais $A_{i} \cap A_{j}=\varnothing$ quando $i \neq j$ temos

que:

$$
P\left(\bigcup_{i=1}^{\infty} A_{i}\right)=\sum_{i=1}^{\infty} P\left(A_{i}\right) \text {. }
$$

Teorema 1.1: Continuidade monótona, presente também na esperança, se $A_{n} \uparrow A$, então $P\left(A_{n}\right) \uparrow 巴(A)$, similarmente se $A_{n} \downarrow A$ então 回 $\left(A_{n}\right) \downarrow$ P (A).

Definição 2.1: Seja uma variável aleatória simples, na forma $X(\omega)=\sum_{i=1}^{n} \mathrm{X}_{\mathrm{i}}{ }^{1}{ }_{\mathrm{Ai}}(\omega), \omega \varepsilon(\Omega)$; no qual $\mathrm{x}_{\mathrm{i}} \varepsilon \mathrm{R}$ são números reais distintos e $\left\{A_{i}: i=1,2, \ldots, n\right\} \subset A$, é uma partição de $\Omega$. Teorema 2.1: Para todo $\mathrm{X}$, uma variável aleatória positiva, existe uma sequência de variáveis aleatórias simples que converge pontualmente para $\mathrm{X}$. Definição 2.2: Esperança como limite de v.a.'s simples:

$\mathrm{E}(\mathrm{X})=\lim _{n \rightarrow \infty} \mathrm{E}\left(\mathrm{X}_{\mathrm{n}}\right)=\lim _{n \rightarrow \infty} \sum_{i=1}^{n} \mathrm{X}_{\mathrm{i}}$ P $\left(\mathrm{A}_{\mathrm{i}}\right)$.

Definição 3.1: Seja uma variável aleatória positiva $X$, dado a $\sigma$-álgebra $G(\subset F)$, é denotada por $E(X \mid G)$ é a unica (९- q.c.) v.a. satisfazendo que $E[X \mid G]$ é

G-mensurável e que para todo $A \varepsilon G$ temos $\int_{A} X d \rho=\int_{A}$
$E[X \mid G] d$. Teorema 3.1: (Teorema de Radon-Nikodym) Seja $(X, F, \mu)$ um espaço de medida, com $\mu$ medida finita. Se $v$ é uma medida finita sobre $F$ absolutamente contínua com respeito a medida $\mu$, então existe uma função mensurável finita f sobre $\mathbf{X}$ tal que $v(E)=\int_{E} f d \mu$ para todo conjunto mensurável $\mathrm{E}$.

Definição 4.1: Um conjunto de v.a.'s que descrevem a evolução de um sistema ao longo do tempo através da filtragem. Teorema 4.1: Dado $(\Omega, F, P)$ um espaço de probabilidade, uma coleção de v.a.'s $\{X(t), t \in T\}$, onde $T \subset R$ é um conjunto de índices. Cada $X(t)$ assume valores em um conjunto $S \subset R$ ( $S$ é o espaço de estados), e $X(t)$ é o estado do processo no tempo t. Assim:

$$
F_{\mathrm{t} 1, \ldots, \mathrm{tn}}\left(\mathrm{X}_{1}, \ldots, \mathrm{x}_{\mathrm{n}}\right)=P\left[\bigcap_{i=1}^{n}\left\{\omega \varepsilon \Omega / \mathrm{X}_{\mathrm{t} 1}(\omega) \leq \mathrm{X}_{\mathrm{i}}\right\}\right] \text {. }
$$

Definição 5.1: Seja um processo estocástico $\{X n, n \geq 0\}$ assumido valores em $S \subset Z$ e descrito por: $X_{n}=i(0$ processo está no estado i no tempo n) então existe uma probabilidade fixada $P_{i j}$ que esteja no estado j no tempo $n+1$. Isto é, admite-se que: $P\left[X_{n+1}=j \mid X_{n}=i, X_{n-1}=\right.$ $\left.\mathrm{i}_{\mathrm{n}-1}, \ldots, \mathrm{X}_{0}=\mathrm{i}_{0}\right]=P^{\mathrm{a}}\left[\mathrm{X}_{\mathrm{n}+1}=\mathrm{j} \mid \mathrm{X}_{\mathrm{n}}=\mathrm{i}\right]=\mathrm{P}_{\mathrm{ij}}$ para quaisquer estados $i_{0}, i_{1}, \ldots, i, j$ e todo $n \geq 0 .\left\{X_{n}, n \geq 0\right\}$.

Teorema 5.1: (Chapman-Kolmogorov):

$$
P\left[X_{m}=j\right]\left[X_{n}=i\right]=\sum_{m=1}^{\infty} P_{m-k}\left[X_{m}, j\right] P_{k-n}\left[i, X_{n}\right]
$$

\section{Conclusões}

Foram desenvolvidos conceitos acerca da construção de uma $\sigma$-álgebra, em seguida definimos a probabilidade via os axiomas de Kolmogorov. Além disso, definimos propriedades da probabilidade e da esperança (integral de Lebesgue), a qual é uma generalização da integral de Riemann. Em seguida, foi caracterizada a esperança condicional, que mostramos que existe e está bem definida via o teorema de Radon-Nikodym. Por fim, analisou-se processos estocásticos com variáveis aleatórias em diferentes estados ao longo do tempo.

[1] Chung, Kai Lai. Markov chains. Berlin: Springer-Verlag, 1967.

[2] Protter, P. E. Stochastic modelling and applied probability, 2005.

[3] Shiryaev, A. N. Probability, volume 95 of graduate texts in mathematics, 1996. 